Methods Between 1 April 2009 and 30 September 2010, metrics data for the GYT: Get Yourself Tested campaign were collected and evaluated from such social media venues as Facebook, Twitter, the GYTnow SMS code, and the GYTnow campaign website. Facebook and Twitter usage were indicators of user engagement with the campaign, and behavioural intentions were measured through use of a STD testing center locator from the GYT website and use of the GYT Short Message Service (SMS) code. A mobile phone user can send a zip code to the SMS service to receive information about local testing centers.

Results Evaluation of the GYT social media efforts allowed campaign organisers to measure campaign reach and engagement, customer sentiment, and intentions to get tested for STDs. The campaign reached over one million people in five national, social media venues. The Facebook site recruited 4177 fans, and the Twitter account had 1719 followers. The testing center locator was used by 64000 people; 51,000 people used the SMS code to locate services. Qualitative data provided additional information about campaign sentiment as well as barriers to participation in the campaign. Analysis of the various metrics also uncovered unexpected issues such as a significant dip in participation in April as a result of a lack of participation by one of the major phone carriers. Conclusions Evaluating social media metrics can provide an in-depth understanding of how well target audiences are being reached, how information and messages resonate with them and how efforts can be improved or changed. Findings from this analysis help illustrate the need to adequately evaluate social media efforts, guide future social media evaluations, and understand audience behaviours when engaging in social media activities. Additional web analytic studies are needed to better understand the impact of social media use for STD prevention.

\section{P2-S8.11 CO-CREATING THE DUTCH SAFE SEX CAMPAIGN 2011: USING SOCIAL MEDIA AND MUNICIPAL HEALTH CENTRES (MHC) TO IMPROVE IMPLEMENTATION INTENTIONS OF CONDOM USE}

doi:10.1136/sextrans-2011-050108.385

F Zimbile, E Fisser. STI Aids The Netherlands, Amsterdam, Netherlands

In the period 2008-2009 the Dutch Safe Sex Campaign (DSSC) effectively improved the attitudes and intentions among young people (18-25 yrs) to maintain condom use in a new relationship until both partners have done a STI test. The positive intentions increased from 50\% (Aug 2008) to 73\% (Nov 2009). However, no changes were seen in self-reported condom use in this period. Theoretical models show that in case of positive intentions a strategy aimed at improving implementation intentions can be effective on behavioural level. In line with this strategy DSSC 2011 is designed to improve implementation intentions in the chain of skills which are relevant for condom use: buying-carrying-communicating about and using condoms.

Method The campaign strategy consists of three stages. In the third stage (Sep 2011) an e-learning module will be launched which will train condom skills and help to concretely plan condom use. The first two stages are especially designed to generate content for the e-learning module in co-creation with the target group members and the MHC. In the first (awareness) stage of the campaign (Feb 2011) members of the target group were invited to report about the campaign. A kick off session was organised for editors of school magazines and popular vloggers (video webloggers). During the kick off the young reporters were facilitated to produce their own safe sex campaign materials. They were also given the opportunity to interview a Dutch celebrity about the campaign topic. The results were used to promote the campaign on
YouTube, Twitter and local school magazines. At the same time discussions and polls about the campaign topic were initiated on several community sites. In the first stage, the campaign also provided a game to the MHC. This game is played in school classes with students of secondary education. The game consists of settings that are relevant for the e-learning module: a drugstore (buying condoms), schoolyard and disco (carrying condoms), bedroom (communicating and using condoms). The MHC officers invited the student to discuss the condom skills in these settings and register the advices that are most relevant to them. These advices will be used as content in the e-learning module. In the second stage (April-June 2011) the target population will be mobilised to participate in the campaign through popular social media. The target group will be asked to reflect on the results of the discussions and polls in the first stage. They will also be invited to send in their ideas for a TV-campaign. The concept of the winner will be used as input for the definite TV-commercial(s) broadcasted in September 2011

Results Almost 200 school magazine reporters and 11 vloggers participated in the first stage of the campaign. More than 80000 people viewed the nine vlogs that were produced in the first week. Conclusion Social media offer good opportunities to co-create safe sex campaigns with the target group.

\section{P2-S8.12 FEASIBILITY OF USING CELL PHONES FOR DAILY DATA COLLECTION WITHIN ADOLESCENT COHORT STUDIES}

doi:10.1136/sextrans-2011-050108.386

C Malotte, A Cutting, S Huettner, P Matson, J Ellen. Johns Hopkins University, Baltimore, USA

Background It is widely recognised that adolescents are a population who encounter frequent changes in their daily lives. In order to capture these important oscillations, daily data are needed to accurately record and track changes in STD associated perceptions and behaviours that adolescents experience.

Methods Adolescent females 16-19 were enrolled in the study from a reproductive healthcare clinic. They are assigned a Palm Centro smartphone, and instructed to make daily diary entries on the phones using Pendragon software continuously for up to 18 months. Field staff meet with the participants every 2 weeks to hot sync the diary data from the phones, which is then compiled into a master database.

Results We examined the number of data points collected over all participants as compared with the costs of the phones, service plans, and person-power needed to complete data collection as well as the subjective opinions of the participants. In an ongoing study, 107 participants have received a phone, and the overall retention rate is $70 \%$. The cost of the cell phone, voice plan and study staff support is approximately $\$ 105$ per month for each participant. Participants have completed an average of 14 diary entries per month, with a total of 9916 collected. All participants who have completed 18 months in the study reported that the diary entry software was somewhat or very easy to use and $63 \%$ indicated that the cell phone was a reason that they remained in the study. Of the 185 phones that were distributed, 84 were reported broken or lost.

Conclusions Utilising this method of data collection has yielded rich data, unlike any currently available in the literature. While the cost of the data collection process may be substantial, there are distinct advantages to the use of cell phones. These benefits include, participant satisfaction, date and time stamped data, an alarm prompt, reduced participant burden, as well as affording interactive and privacy advantages similar to ACASI technology. There are several ways to tailor the collection process so that it may be accessible to a 
wider variety of studies. Greater use of this technology should be an area of interest in further adolescent STD research.

\section{Social and behavioural aspects of prevention poster session 9: Women \\ P2-S9.01 THE IMPACT OF CUSTOMS AND SEXUAL PRACTICES ON YOUNG MAASAI WOMEN'S ABILITY TO NEGOTIATE THEIR SEXUAL AND REPRODUCTIVE HEALTH IN RELATION TO HIV AND AIDS IN LOITOKITOK, KENYA}

doi:10.1136/sextrans-2011-050108.387

J N Matogo. eMentoring Africa, NRB, Kenya

This research investigated the ways in which Maasai culture, as practiced in a rural and relatively isolated area of Kenya, impacted upon the transmission of HIV/AIDS. The author focused her field research on women aged $16-25$, and a range of cultural practices contributing to and influenced by gender roles in Maasai society. Local practice has been addressed and recommendations made in relation to the position of the Maasai community in the wider political economy and Kenya's ethnos cape.

The study also investigated strategies the women considered appropriate, practical and effective to cope with these risks. The field study was conducted in the Loitokitok district of Kenya.

Methodology The research is within a qualitative paradigm. Choice of methodology was mainly based on ethic consideration of research within cultural context of Maasai Indigenous people. Data was generated through use of focus group discussions, semi structured interviews and informal observation methods. An extensive review of the literature was also conducted. The influence of gender based customs and practices are highlighted in a number of scholarly works, Governmental and non-governmental documents with regard to women's vulnerability to Sexually Transmitted Infections (STIs).

The researcher maintained an "insider-outsider" position and a participatory role in order to try to identify the current state of Maasai women's reproductive health at the grass roots level.

Findings Research findings have found that there is a challenge in young pastoralist women's reproductive health autonomy. Their risk of STIs especially HIV infection is strongly determined by cultural and sexual practices that are gender related.

Existing customs and practices tend to be more repressive to the women's autonomy in sexual health matters as men hold power in most important roles in society.

Conclusions The study does not call for cultural change or reformation of traditional culture within the Maasai community; rather it appeals for transformation of customs and practices that cause harm on women's reproductive health. Valuable cultural practices and intentions that recognise women and celebrate their womanhood should be encouraged in a way that is not physically or psychologically daunting experience on their wellbeing.

To this end, the understanding and knowledge of the Maasai worldview is critical to the intervention of Maasai women's reproductive health rights. A more cultural approach to Maasai women's reproductive health is suggested to be more effective. In relation to the adoption of STI prevention measures, Maasai culture plays a key role towards identification of preventive measures and strategies.

\section{P2-S9.02 SYPHILIS AND PREGNANCY: SOCIAL PORTRAIT OF THE DISEASE IN BELARUS}

doi:10.1136/sextrans-2011-050108.388

I Tsikhanouskaya. Vitebsk State Medical University, Vitebsk, Belarus

According to the statistical data in Belarus pregnant women made of $8-17 \%$ of all women with confirmed syphilitic infection. About
$15 \%$ women have primary syphilis, 30\%-secondary, and $55 \%-$ latent syphilis. Aim of study. Study the social structure and concurrent pathology in pregnant women with confirmed syphilitic infection in Belarus.

Methods 95 case histories of pregnant women with confirmed diagnosis of syphilitic infection where analysed during 2003-2008. Middle age of women was $25.5 \pm 51$ years.

Results Middle gestational period when syphilitic infection was registered was $21.5 \pm 20.4$ weeks (in 1-12 weeks-in $10,5 \%$ of women; in 13-24 weeks-in 56.8\%, in 25-36 weeks-in 30.5\%, and in 37-40 weeks-in 2.2\%). Concurrent STI where registered in $66.3 \%$ of women: trichomoniasis in $11.1 \%$, urogenital candidiasis-in $25,4 \%$, urogenital chlamydiasis-in $12.7 \%$, mixed infection (chlamydia and ureoplasma) in $4.8 \%$. Anaemia was registered in $25.1 \%$ of cases, fetoplacental insufficiency-in $7.1 \%$ of cases. From all women $67.3 \%$ where not officially married and their partners where not checked up as far as they where working outside Belarus. Only 8 women (8.4\%) mentioned occasional sexual contacts. Unemployed women made of $52.6 \%$ of studied patients. Majority of employed women $(64 \%)$ worked as unskilled workers. Others where academics-6.67\%, medical professions-8.89\%, students-8.89\%, others-6.67\%.

Conclusion Syphilitic infection in Belarus is detected quite late (usually 13-36 weeks). High percentage of pregnant women in Belarus are women with low social status and poor education.

\section{P2-S9.03 THE SEX LIVES OF EMERGENCY CONTRACEPTION USERS IN THE USA, 2006-2008}

doi:10.1136/sextrans-2011-050108.389

M Habel, J S Leichliter. CDC, Atlanta, USA

Background Previous research indicates that emergency contraception (EC) users are less likely to have visited a gynaecologist in the past 12 months, and are more likely to report ever having an STI compared to non-users; however, studies examining this unique population have been outside of the USA Given FDA-licensure of EC behind-thecounter, this is the first US study to use a nationally representative sample of reproductive aged women (15-44) to explore whether EC users represent a missed opportunity for STI counselling and screening. Methods Data were collected through in-person interviews and through audio computer-assisted self-interview. Using a sample of 7356 women, sexual behaviour variables for which there were significant bivariate differences $(p<0.10)$ for lifetime EC users were examined in a multiple logistic regression model controlling for demographics often associated with sexual behaviour including age, race/ethnicity, marital status, poverty level, and geographic location. Results Overall 10\% (704) of the sample had ever used EC; less than $3 \%$ had used it within the past 12 months. Most women had only used EC once (62\%). Primary reasons for use were not using a birth control method (46\%) and worry that birth control would not work $(42 \%)$. Most EC users had received EC from a family planning clinic (51\%). More EC users obtained EC from a drug store drug store $(23 \%)$ than a private doctor's office (17\%); most received EC without a prescription (69\%). Demographic factors associated with lifetime EC use included: age 20-24/25-29 years (AORs=3.3; 2.4), never married (2.1), income $150 \%$ above the poverty level (1.6), and living in an urban-suburban area (1.6). Lifetime EC users were almost twice as likely to have had $>4$ lifetime partners. In bivariate analyses lifetime EC use was associated with receiving STI services in the past 12 months and having had a pap, but in the model became insignificant.

Conclusions Contrary to previous findings, EC users were no more likely than non-users to have received STI counselling or screening, despite greater numbers of sex partners. However, with licensure of behind-the-counter ECs, this research indicates that some women are accessing ECs without a prescription at drug stores rather than a provider's office, representing a missed opportunity for screening. These 